\title{
Biosynthetic Origins of C-P Bond Containing Tripeptide K-26
}

Ioanna Ntai ${ }^{1}$, M. Lisa Manier ${ }^{2}$, David L. Hachey², Brian O. Bachmann ${ }^{1 *}$

Vanderbilt Institute of Chemical Biology, ${ }^{1}$ Department of Chemistry,

${ }^{2}$ Department of Biochemistry, Mass Spectrometry Research Center.

\section{Supplementary Material}


Fermentation. The K-26 producing strain (Actinomycetes sp K26) was obtained from the Agricultural Research Service (NRRL 12379). The production protocol suggested by Yamato et $\mathrm{al}^{1}$ was followed with minor modifications. The seed medium consisted of dextrose, $0.1 \mathrm{~g} / \mathrm{L}$; Difco soluble starch, 0.1g/L; Bacto beef extract, 0.05g/L; Bacto yeast extract, 0.05g/L; Bacto tryptone, $0.05 \mathrm{~g} / \mathrm{L}$; and $\mathrm{CaCO}_{3}, 0.02 \mathrm{~g} / \mathrm{L}$ dissolved in distilled water and was adjusted to $\mathrm{pH} 7.2$ before autoclaving. Fermentation was initiated by aseptically inoculating a loopful of K-26 grown on an agar plate into a sterile 50-mL Falcon tube containing $10 \mathrm{~mL}$ seed medium (Phase I). The Falcon tube was incubated for $10-12$ days at $28^{\circ} \mathrm{C}$ in a shaker incubator. In the second phase, $3 \mathrm{~mL}$ of the phase I seed culture were transferred into a $300-\mathrm{mL}$ flask containing $30 \mathrm{~mL}$ of seed medium. The flask was incubated for $3-4$ days at $28^{\circ} \mathrm{C}$ in a shaker incubator. In phase III, $30 \mathrm{~mL}$ of phase II seed culture were transferred into a 3000-mL Fernbach flask containing $300 \mathrm{~mL}$ production medium. The flask was incubated for $5-6$ days at $28^{\circ} \mathrm{C}$ in a shaker incubator. The production medium contained Difco soluble starch, $0.4 \mathrm{~g} / \mathrm{L}$; soy bean meal (Wild Oat), 0.3g/L; corn steep liquor (Sigma), 0.05g/L; $\mathrm{K}_{2} \mathrm{HPO}_{4}, 5 \mathrm{mg} / \mathrm{L} ; \mathrm{MgSO}_{4}, 2.4 \mathrm{mg} / \mathrm{mL}$; $\mathrm{KCl}, 3 \mathrm{mg} / \mathrm{L}$; and $\mathrm{CaCO}_{3}, 0.03 \mathrm{~g} / \mathrm{L}$ dissolved in distilled water and was adjusted to $\mathrm{pH} 7.8$ before autoclaving.

Chemicals. All stable isotopically labeled precursors were purchased from Cambridge Isotope Laboratories.

Pulse Feeding Experiments. One millimolar amounts (based on the culture volume) of each precursor were dissolved in $5 \mathrm{~mL}$ water and administered separately to phase III culture through a sterile syringe filter in every 24 hours for 5 days.

K-26 purification. The production culture was centrifuged and the supernatant was acidified to $\mathrm{pH}$ 3.00. To one liter of supernatant were added thirty grams of the hydrophobic 
resin Diaion HP-20 (activated by stirring with methanol and rinsing with water) and the suspension was stirred for 30 minutes. The resin was filtered and washed with water (2x30mL). The washed resin beads were placed in a 50\% methanol-50\% water solution and stirred for 10 min. The suspension was then filtered, washed with 50\% methanol (2x30 mL), and discarded. The filtrate was concentrated to $40 \mathrm{~mL}$ and neutralized. K-26 was further purified using Millipore Ultra 5000 MWCO centrifugal filter devices and centrifuging at $3450 \mathrm{~g}$ for 30 minutes. The filtrate was concentrated to ca. one $\mathrm{mL}$ by rotary evaporation.

ACE assay. To each well of a 96-well plate were added $100 \mu \mathrm{L}$ FAPGG reagent, 60 $\mu \mathrm{L}$ ACE extract and $60 \mu \mathrm{L}$ sample. The FAPGG reagent consisted of $3.4 \mathrm{mg}$ furanacryloyl Phe-Gly-Gly, $0.5 \mathrm{~mL}$ Tris buffer (1M, pH 8.0), $6 \mathrm{~mL} \mathrm{NaCl} \mathrm{(1M)} \mathrm{solution,} \mathrm{and} 3.5 \mathrm{~mL} \mathrm{H}_{2} \mathrm{O}$. ACE was extracted from rabbit lung acetone powder by suspending $0.2 \mathrm{~g}$ in $6 \mathrm{~mL}$ potassium phosphate (50 mM, pH 8.3) buffer followed by centrifuging at $1400 \mathrm{rpm}$ for 30 minutes. The supernatant is the ACE extract mentioned above. Absorbance readings were made with a Spectramax UV/Vis spectrometer at $340 \mathrm{~nm}$ every 10 seconds over a period of 5 minutes.

\section{Mass spectrometry}

General. Mass spectrometry was performed using ThermoFinnigan (San Jose, CA) TSQ ${ }^{\circledR}$ Quantum triple quadrupole mass spectrometer equipped with a standard electrospray ionization source outfitted with a 100- $\mu$ m I.D. deactivated fused Si capillary. Data acquisition and spectral analysis were conducted with Xcalibur ${ }^{\mathrm{TM}}$ Software, version 1.3, from ThermoFinnigan (San Jose, CA), on a Dell Optiplex GX270 computer running the Microsoft ${ }^{\circledR}$ Windows 2000 operating system. The source spray head was oriented at an angle of $90^{\circ}$ to the ion-transfer-tube. Nitrogen was used for both the sheath and auxiliary gas. The sheath and auxiliary gases were set to 33 and 14 (arbitrary units) respectively. Samples were introduced by 
HPLC. A Surveyor ${ }^{\circledR}$ Autosampler and a Surveyor ${ }^{\circledR}$ MS Pump from ThermoFinnigan (San Jose, CA) were used. The injection volume was $10 \mu \mathrm{L}$. K-26 was separated from co-metabolites using a Jupiter ${ }^{\mathrm{TM}}$ minibore $5 \mu \mathrm{m}$ C18 column $(2.0 \mathrm{~mm} \times 15 \mathrm{~cm})$ with a linear water-acetonitrile gradient (ranging from 95:5 to 5:95 $\mathrm{H}_{2} \mathrm{O}: \mathrm{CH}_{3} \mathrm{CN}$ ) containing $10 \mathrm{mM}$ ammonium acetate. The flow rate was $0.2 \mathrm{~mL} / \mathrm{min}$.

Comparison of biogenic K-26 to synthetically prepared sample. Synthetic K-26 was prepared, with minor modification, according to the procedure of: Kasai, M.; Yoshida, N.; Hirayama, N.; Shirahata, K. Symposium Papers, The 27th Symposium on the Chemistry of Natural Products 1985, 577. (Data to be published elsewhere). Biogenic K-26 was identical to synthetic sample as shown in figure 1.
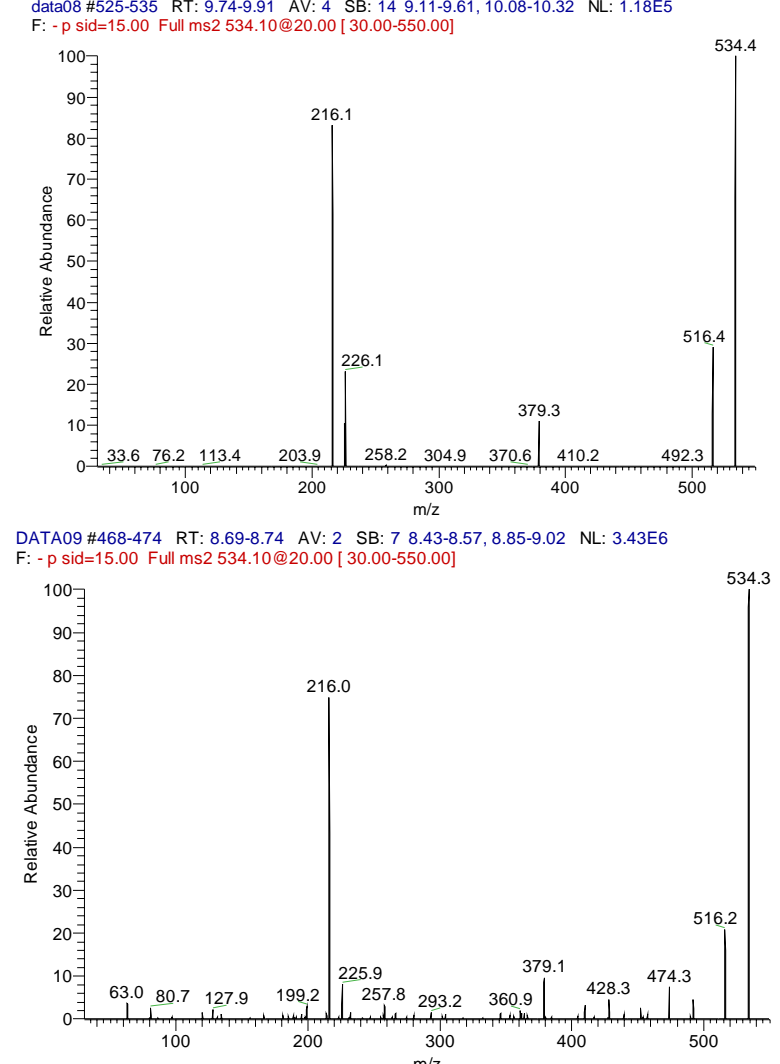

Figure 1. $\mathrm{MS}^{2}$ of 534 ion of synthetic (above) versus biogenic (below) K-26. 
Ring- $\boldsymbol{d}_{4}$-tyrosine. The mass spectrometer was operated in the positive ion mode and the electrospray needle was maintained at $4200 \mathrm{~V}$. The ion transfer tube was operated at $35 \mathrm{~V}$ and $300^{\circ} \mathrm{C}$. The tube lens voltage was set to $131 \mathrm{~V}$. Source CID (offset voltage between skimmer and the first ion quide, Q00) was used at $15 \mathrm{~V}$. The selected reaction monitoring (SRM) mode was used. Ions were collisionally activated with argon at an indicated pressure of 1.5mT. The mass-spectral resolution was set to a peak width (full width at half maximum, FWHM) of $0.70 \mathrm{u}$ and $0.70 \mathrm{u}$ for precursor and product ions respectively. Mass transitions at the specified collision energy (m/z 536 $\rightarrow 218 ; 20 \mathrm{eV}),(\mathrm{m} / \mathrm{z} 540 \rightarrow 218 ; 20 \mathrm{eV})$, and $(\mathrm{m} / \mathrm{z} 540 \rightarrow 222$; $20 \mathrm{eV}$ ) were monitored for unenriched $\mathrm{K}-26$, enrichment in the central tyrosine and enrichment in AHEP, respectively. The scan width for product ions was $1.000 \mathrm{u}$ and the cycle time for each ion was 0.25 seconds. The electron multiplier gain was set to $2 \times 10^{6}$. Data were acquired in profile mode. The resulting chromatogram is illustrated in figure 2 .

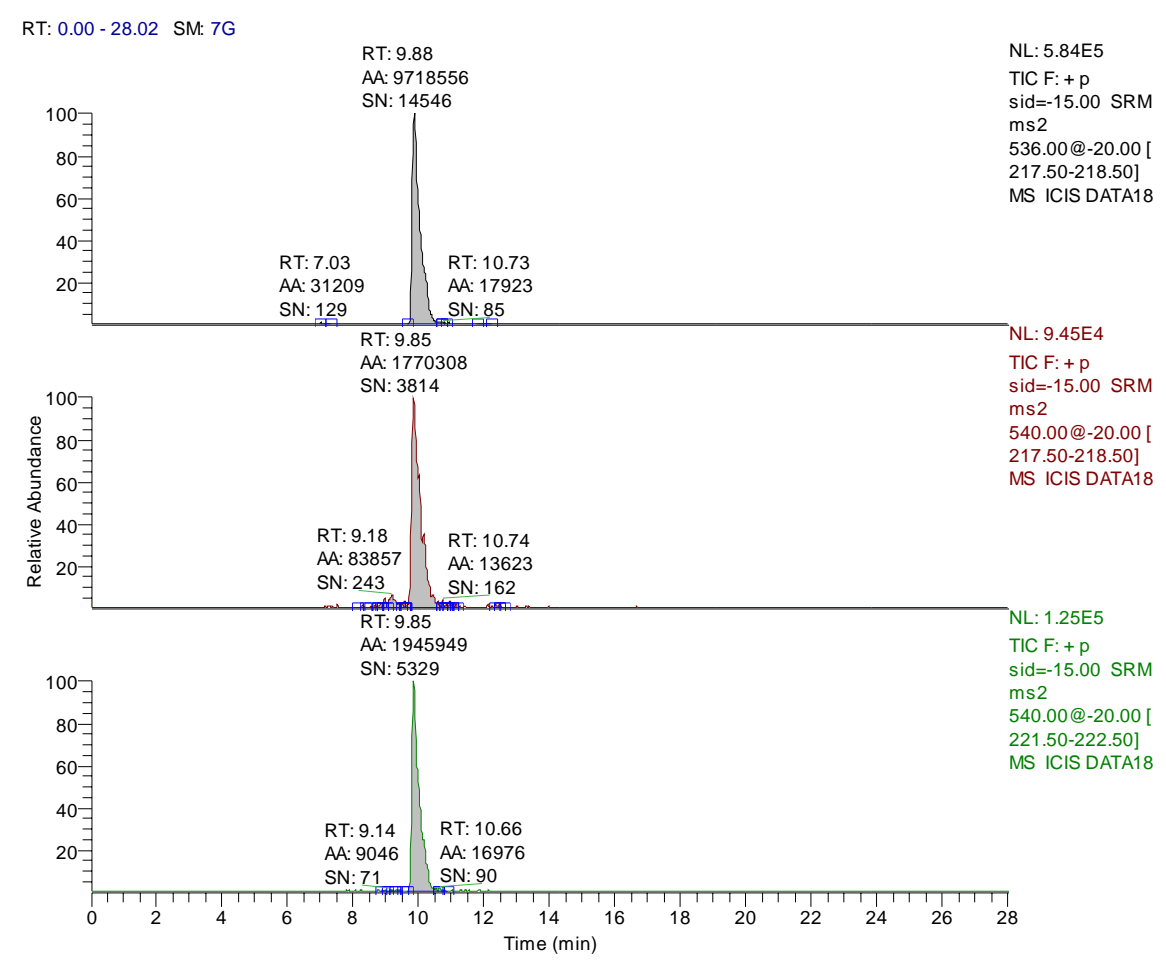

Figure 2. Chromatograms used for the calculation of ring- $d_{4}$-tyrosine incorporation 
Table 1. Calculation of ring- $d_{4}$-tyrosine incorporation

\begin{tabular}{ccccccccc}
\hline Transition & \multicolumn{3}{c}{ Area } & & \multicolumn{5}{c}{ Isotope Distribution } \\
& $\mathbf{1}$ & $\mathbf{2}$ & $\mathbf{3}$ & $\mathbf{1}$ & $\mathbf{2}$ & $\mathbf{3}$ & average & SD \\
$536-->218$ & 9701469 & 9674024 & 9736478 & 100 & 100 & 100 & 100 & 0 \\
$540-->218$ & 1719261 & 1658059 & 1755359 & 17.72 & 17.13 & 18.03 & 17.63 & 0.45 \\
$540-->222$ & 1983006 & 1954870 & 1962925 & 20.44 & 20.21 & 20.16 & 20.27 & 0.15 \\
\hline
\end{tabular}

The isotope distribution was calculated by assigning the $536 \rightarrow 218$ peak an area of 100 and normalizing the other peaks accordingly.

${ }^{15} \mathrm{~N}$-tyrosine. The mass spectrometer was operated in the negative ion mode and the electrospray needle was maintained at $4200 \mathrm{~V}$. The ion transfer tube was operated at $-35 \mathrm{~V}$ and $350^{\circ} \mathrm{C}$. The tube lens voltage was set to $-150 \mathrm{~V}$. Source CID (offset voltage between skimmer and the first ion quide, Q00) was used at 15V. The selected reaction monitoring (SRM) mode was used. Ions were collisionally activated with argon at an indicated pressure of $1.4 \mathrm{mT}$. The mass-spectral resolution was set to a peak width (full width at half maximum, FWHM) of $0.50 \mathrm{u}$ and $0.50 \mathrm{u}$ for precursor and product ions respectively. Mass transitions at the specified collision energy ( $\mathrm{m} / \mathrm{z} 534 \rightarrow 216 ; 35 \mathrm{eV})$, and $(\mathrm{m} / \mathrm{z} 535 \rightarrow 217 ; 35 \mathrm{eV})$ were monitored for AHEP and AHEP+1, respectively. Transitions (m/z 534 $\rightarrow 216 ; 35 \mathrm{eV}),(\mathrm{m} / \mathrm{z} 535 \rightarrow 216 ; 35 \mathrm{eV})$, and $(\mathrm{m} / \mathrm{z}$ 536 $\rightarrow 216$; 35eV) were monitored for $N$-Ac-Ile-Tyr, $N$-Ac-Ile-Tyr +1 , and $N$-Ac-IleTyr+2, respectively. Transitions (m/z 534 $\rightarrow 379 ; 35 \mathrm{eV}),(\mathrm{m} / \mathrm{z} 535 \rightarrow 380 ; 35 \mathrm{eV})$, and $(\mathrm{m} / \mathrm{z}$ $536 \rightarrow 381 ; 35 \mathrm{eV}$ ) were monitored for Tyr-AHEP, Tyr-AHEP+1, and Tyr-AHEP+2, respectively. Finally, transitions $(\mathrm{m} / \mathrm{z} 534 \rightarrow 379 ; 35 \mathrm{eV}),(\mathrm{m} / \mathrm{z} 535 \rightarrow 379 ; 35 \mathrm{eV})$, and $(\mathrm{m} / \mathrm{z}$ $536 \rightarrow 379 ; 35 \mathrm{eV}$ ) were monitored for $N$-Ac-Ile, $N$-Ac-Ile +1 , and $N$-Ac-Ile +2 , respectively. The scan width for product ions was $1.000 \mathrm{u}$ and the cycle time for each ion was 0.15 seconds. The electron multiplier gain was set to $2 \times 10^{6}$. Data were acquired in profile mode. The resulting 
chromatograms are illustrated in figures 3,4 and 5. Both a ${ }^{15} \mathrm{~N}$-tyrosine enriched sample and an unenriched sample were scanned. The unenriched sample was used to create theoretical curves of ratios of mass isotopomer abundance in K-26 to correct the isotopomer distribution of the enriched sample.

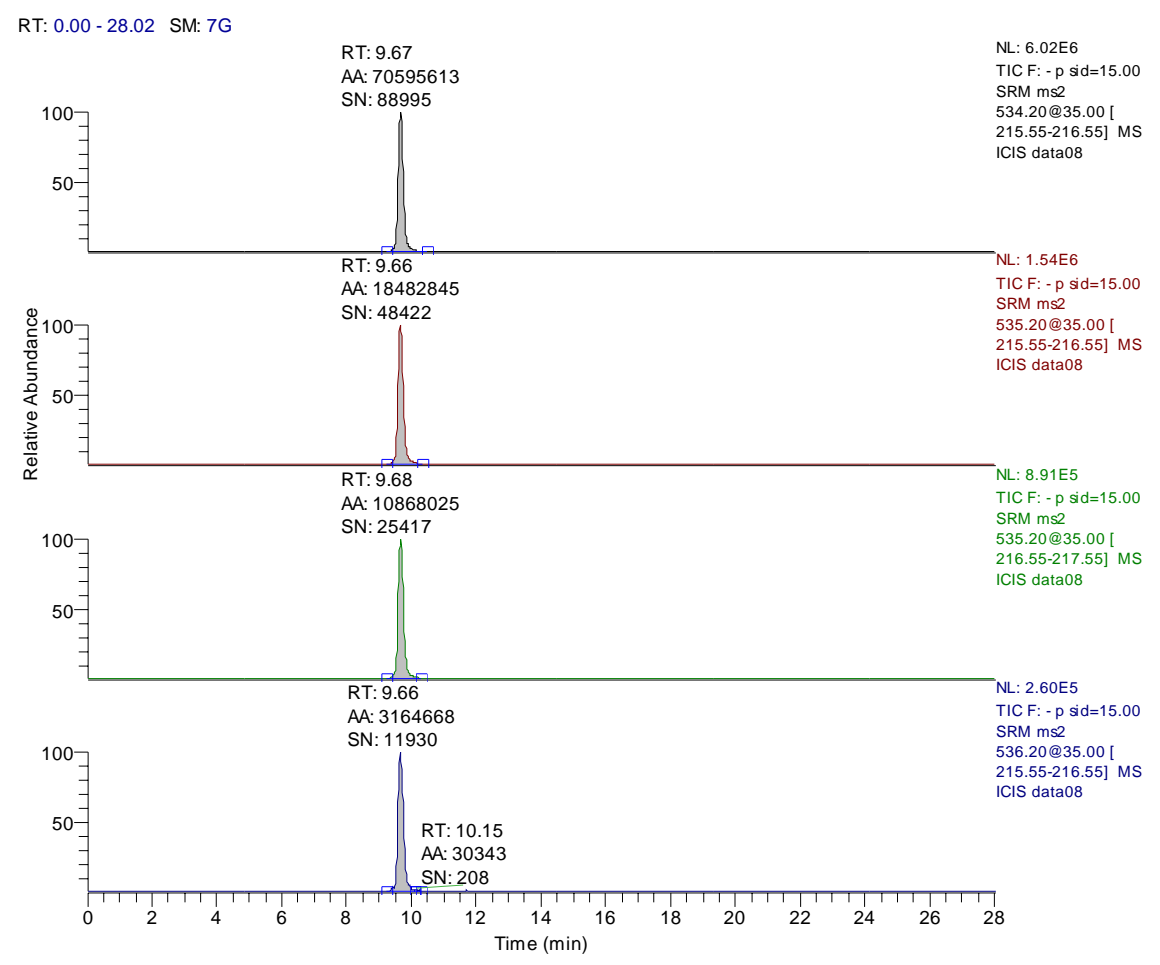

Figure 3. Chromatograms used in the calculation of ${ }^{15} \mathrm{~N}$-tyrosine incorporation in AHEP 


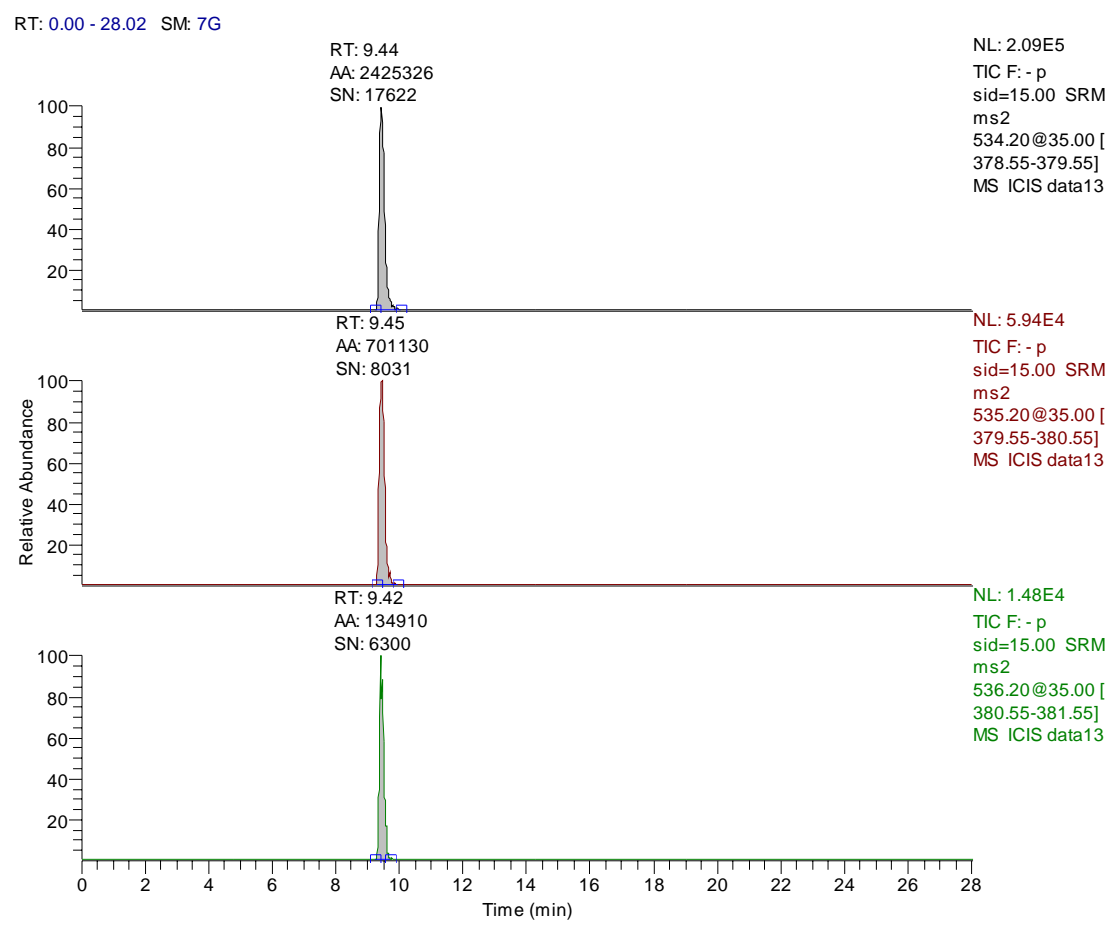

Figure 4. Chromatograms used in the calculation of ${ }^{15} \mathrm{~N}$-tyrosine incorporation in Tyr-AHEP

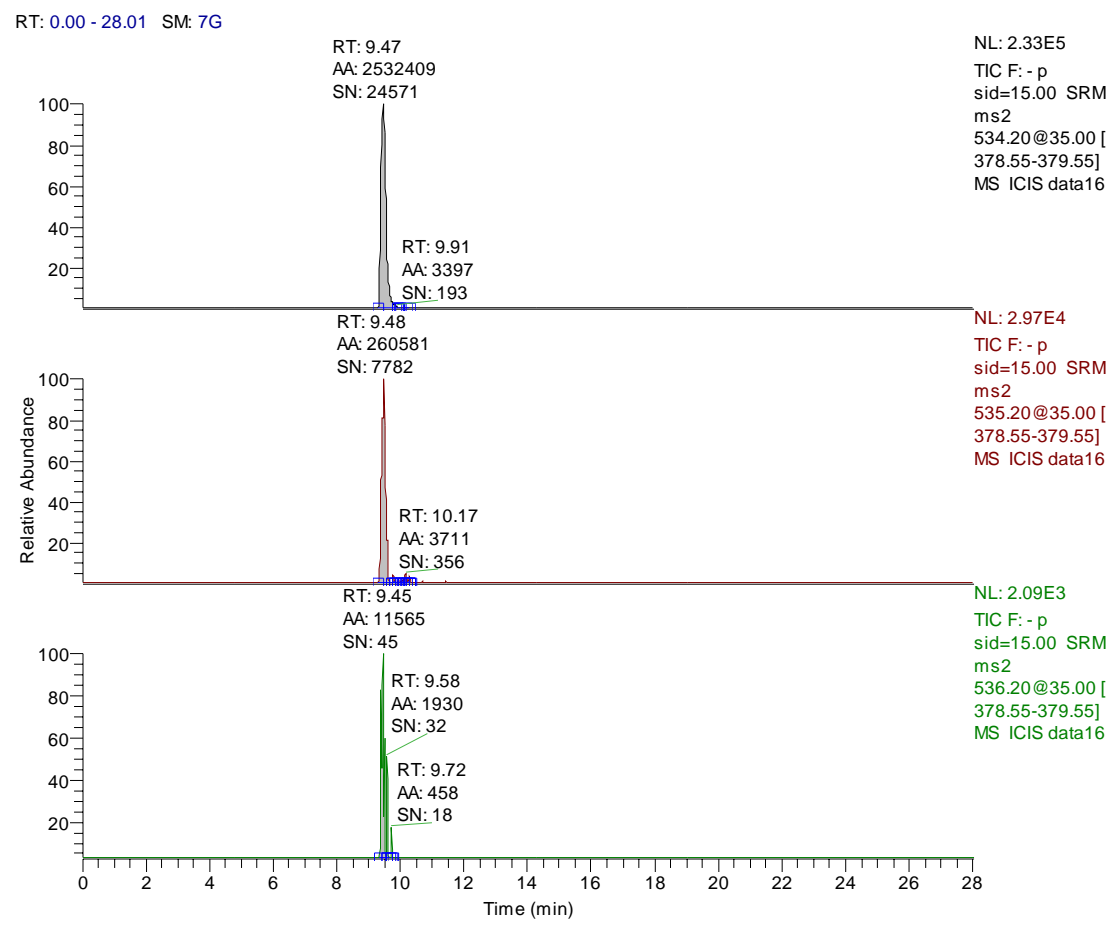

Figure 5. Chromatograms used in the calculation of ${ }^{15} \mathrm{~N}$-tyrosine incorporation in N-Ac-IleTyr 
Calculation of ${ }^{15} \mathrm{~N}$ enrichment example. The tabulated numbers below (Calculation 1-4) resulted from the following calculations. Since all the calculations (1-4) are similar, the deconvolution of tandem MS data in order to determine ${ }^{15} \mathrm{~N}$ enrichment in Tyr-AHEP (Calculation 4) exemplifies the method. In this example, the theoretical isotope matrix was constructed using the relative mass spectral ion intensities of transitions $534 \rightarrow 379,535 \rightarrow 380$, and $536 \rightarrow 381$ measured for an unenriched sample. In the absence of a pure standard for ${ }^{15} \mathrm{~N}_{1}$ $\mathrm{K}-26$ and ${ }^{15} \mathrm{~N}_{2}-\mathrm{K}-26$, we assumed that the theoretical distribution of unenriched $\mathrm{K}-26$ would be as follows.

\begin{tabular}{|c|c|c|c|}
\hline \multicolumn{4}{|c|}{ Theoretical Isotope Ratio (x100) } \\
\hline $\mathrm{m} / \mathrm{z}$ & ${ }^{15} \mathrm{~N}_{0}-\mathrm{PA}$ & ${ }^{15} \mathrm{~N}_{1}-\mathrm{PA}$ & ${ }^{15} \mathrm{~N}_{2}-\mathrm{PA}$ \\
\hline $534->379$ & 100.00 & 0.00 & 0.00 \\
\hline $535->380$ & 16.89 & 100.00 & 0.00 \\
\hline $536->381$ & 1.86 & 16.53 & 100.00 \\
\hline & & 1.86 & 16.17 \\
\hline & & & 1.86 \\
\hline
\end{tabular}

The mole fraction matrix was obtained by normalizing the relative ion intensities to the sum of the ion intensities for each species $\left({ }^{15} \mathrm{~N}_{0},{ }^{15} \mathrm{~N}_{1}\right.$, and ${ }^{15} \mathrm{~N}_{2}$, the tracer substances). The mole fraction matrix was transposed resulting in the isotope design matrix, in order that the final result would be along the same row instead of down the same column. This set of normalized mass spectral ion intensities can be used as a set of fixed coefficients in a system of linear equations that describe the relative ion abundances of the different tracers. The general form of the equation is

$$
\mathrm{I}_{\mathrm{j}}=\mathrm{A}_{\mathrm{i}, \mathrm{j}} \times \mathrm{B}_{\mathrm{i}} \quad \text { equation } 1
$$

Where $I_{j}$ is the intensity of transition $j, A_{i, j}$ is the normalized ion abundance of tracer substance $\mathrm{i}$ during transition $\mathrm{j}$ (isotope design matrix), and $\mathrm{B}_{\mathrm{i}}$ is the unknown mole fraction of tracer 
substance i. The system has to include one equation for each tracer and each equation must contain one term for each tracer. This results in the following set of linear equations:

$$
\begin{aligned}
& \mathrm{I}_{534 \rightarrow 379}=84.213 \mathrm{~B}_{15 \mathrm{~N} 0}+14.219 \mathrm{~B}_{15 \mathrm{~N} 1}+1.568 \mathrm{~B}_{15 \mathrm{~N} 2} \\
& \mathrm{I}_{535 \rightarrow 380}=0.000 \mathrm{~B}_{15 \mathrm{~N} 0}+84.467 \mathrm{~B}_{15 \mathrm{~N} 1}+13.962 \mathrm{~B}_{15 \mathrm{~N} 2} \\
& \mathrm{I}_{536 \rightarrow 381}=0.000 \mathrm{~B}_{15 \mathrm{~N} 0}+0.000 \mathrm{~B}_{15 \mathrm{~N} 1}+84.724 \mathrm{~B}_{15 \mathrm{~N} 2}
\end{aligned}
$$

In order to solve this set of linear equations, the pseudoinverse matrix was calculated according to the following equation.

$$
P=\left(A^{T} \times A\right)^{-1} \times A^{T}
$$

equation 2

Where $\mathrm{P}$ is the pseudoinverse matrix, $\mathrm{A}$ is the isotope design matrix and $\mathrm{A}^{\mathrm{T}}$ is the transpose of A. Multiplying $\mathrm{P}$ with the measured isotope ratio matrix (normalized measured ion intensities) gives the corrected isotopic abundances and ratio. This calculation was performed for both unenriched and enriched samples to check for errors in the method. The corrected isotopic abundances were normalized to 100 . The unenriched samples had corrected tracer: tracee ratio of 100:0:0 for ${ }^{15} \mathrm{~N}_{0}:{ }^{15} \mathrm{~N}_{1}:{ }^{15} \mathrm{~N}_{2}$ while the enriched sample had a ratio of 100:12.49:1.43, clearly indicating ${ }^{15} \mathrm{~N}$ enrichment. Simulated isotopic distributions based on least squares fitting of isotopomer data in Calculations 1-4 are shown in figure 6. 
Calculation 1: Presence of $15 \mathrm{~N}$ in the $\mathrm{N}$-terminal Isoleucine or the central Tyrosine Note: Neutral loss isotopic enrichment model

\begin{tabular}{|c|c|c|c|c|c|c|c|}
\hline \multicolumn{4}{|c|}{ Theoretical Isotope Ratio (x100) } & \multicolumn{4}{|c|}{ Mole Fraction (x100) } \\
\hline $\mathrm{m} / \mathrm{z}$ & ${ }^{15} \mathrm{~N}_{0}-\mathrm{PA}$ & ${ }^{15} \mathrm{~N}_{1}$-PA & ${ }^{15} \mathrm{~N}_{2}-\mathrm{PA}$ & $\mathrm{m} / \mathrm{z}$ & ${ }^{15} \mathrm{~N}_{0}$-PA & ${ }^{15} \mathrm{~N}_{1}-\mathrm{PA}$ & ${ }^{15} \mathrm{~N}_{2}-\mathrm{PA}$ \\
\hline $534->216$ & 100.00 & 0.00 & 0.00 & $534->216$ & 83.13 & 0.00 & 0.00 \\
\hline $535->216$ & 17.88 & 100.00 & 0.00 & $535->216$ & 14.87 & 83.39 & 0.00 \\
\hline $536->216$ & 2.40 & $\begin{array}{r}17.52 \\
2.40\end{array}$ & $\begin{array}{r}100.00 \\
17.16 \\
2.40\end{array}$ & $536->216$ & 2.00 & 14.61 & 83.64 \\
\hline
\end{tabular}

\begin{tabular}{|c|c|c|c|c|c|c|c|}
\hline & \multicolumn{3}{|c|}{ Isotope Design Matrix } & & \multicolumn{3}{|c|}{ Pseudoinverse Matrix } \\
\hline & ${ }^{15} \mathrm{~N}_{0}$-PA & ${ }^{15} \mathrm{~N}_{1}$-PA & ${ }^{15} \mathrm{~N}_{2}-\mathrm{PA}$ & & ${ }^{15} \mathrm{~N}_{0}$-PA & ${ }^{15} \mathrm{~N}_{1}$-PA & ${ }^{15} \mathrm{~N}_{2}-\mathrm{PA}$ \\
\hline $534->216$ & 83.135 & 14.866 & 1.999 & $534->216$ & 0.0120 & -0.0021 & 0.0001 \\
\hline $535->216$ & 0.000 & 83.389 & 14.610 & $535->216$ & 0.0000 & 0.0120 & -0.0021 \\
\hline $536->216$ & 0.000 & 0.000 & 83.640 & $536->216$ & 0.0000 & 0.0000 & 0.0120 \\
\hline
\end{tabular}

Sample Measured Isotope Ratio (x100)

\begin{tabular}{|c|c|c|c|}
\hline & ${ }^{15} \mathrm{~N}_{0}-\mathrm{PA}$ & ${ }^{15} \mathrm{~N}_{1}-\mathrm{PA}$ & ${ }^{15} \mathrm{~N}_{2}-\mathrm{PA}$ \\
\hline 1 & 100.000 & 17.831 & 2.370 \\
\hline 2 & 100.000 & 17.823 & 2.427 \\
\hline 3 & 100.000 & 17.992 & 2.416 \\
\hline Mean & 100.000 & 17.882 & 2.405 \\
\hline S.D. & 0.000 & 0.095 & 0.030 \\
\hline 1 & 100.000 & 26.970 & 4.595 \\
\hline 2 & 100.000 & 26.181 & 4.526 \\
\hline 3 & 100.000 & 26.848 & 4.421 \\
\hline Mean & 100.000 & 26.666 & 4.514 \\
\hline S.D. & 0.000 & 0.424 & 0.088 \\
\hline
\end{tabular}

Corrected, Tracer:Tracee Ratio (x100)

\begin{tabular}{lrrr} 
& ${ }^{15} \mathrm{~N}_{0}$-PA & ${ }^{15} \mathrm{~N}_{1}$-PA & ${ }^{15} \mathrm{~N}_{2}$-PA \\
\cline { 2 - 4 } $\mathbf{1}$ & 100.00 & -0.05 & -0.03 \\
$\mathbf{2}$ & 100.00 & -0.06 & 0.03 \\
$\mathbf{3}$ & 100.00 & 0.11 & -0.01 \\
Mean & 100.00 & 0.00 & 0.00 \\
S.D. & 0.00 & 0.09 & 0.03 \\
& & & \\
$\mathbf{1}$ & 100.00 & 9.06 & 0.60 \\
$\mathbf{2}$ & 100.00 & 8.27 & 0.66 \\
$\mathbf{3}$ & 100.00 & 8.94 & 0.44 \\
Mean & 100.00 & 8.76 & 0.57 \\
\hline S.D. & 0.00 & 0.42 & 0.11
\end{tabular}

Corrected Isotopic Abundances \& Ratio

\begin{tabular}{lrr} 
[M] & \multicolumn{1}{c}{$[\mathbf{M}+1]$} & \multicolumn{1}{c}{$[\mathbf{M}+2]$} \\
1.203 & -0.001 & 0.000 \\
1.203 & -0.001 & 0.000 \\
1.203 & 0.001 & 0.000 \\
1.203 & 0.109 & 0.007 \\
1.203 & 0.100 & 0.008 \\
1.203 & 0.108 & 0.005
\end{tabular}


Calculation 2: Presence of $15 \mathrm{~N}$ in the AHEP moiety Note: Charged residue isotopic enrichment model

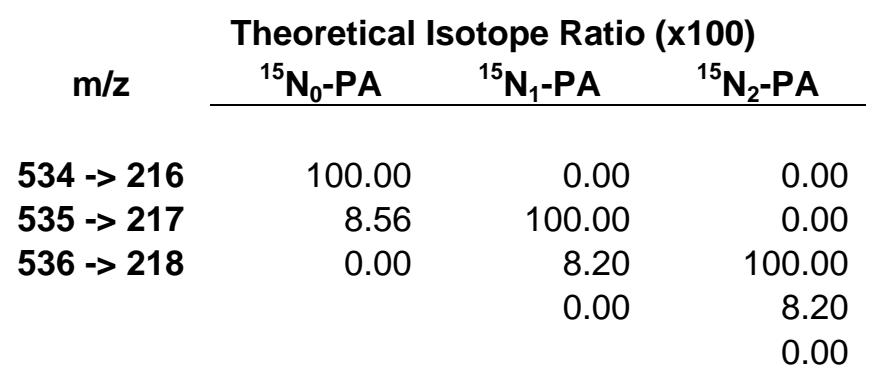

Isotope Design Matrix

\begin{tabular}{rrrr} 
& ${ }^{15} \mathrm{~N}_{0}-\mathrm{PA}$ & ${ }^{15} \mathrm{~N}_{1}$-PA & ${ }^{15} \mathrm{~N}_{2}$-PA \\
\cline { 2 - 4 } $\mathbf{5 3 4}->\mathbf{2 1 6}$ & 92.115 & 7.885 & 0.000 \\
$\mathbf{5 3 5}$-> 217 & 0.000 & 92.421 & 7.579 \\
$\mathbf{5 3 6}$-> 218 & 0.000 & 0.000 & 92.421
\end{tabular}

Sample Measured Isotope Ratio (x100)

\begin{tabular}{lrrr} 
& ${ }^{15} \mathrm{~N}_{0}$-PA & ${ }^{15} \mathrm{~N}_{\mathbf{1}}$-PA & ${ }^{15} \mathrm{~N}_{\mathbf{2}}$-PA \\
\cline { 2 - 4 } $\mathbf{1}$ & 100.000 & 8.509 & 0.000 \\
$\mathbf{2}$ & 100.000 & 8.656 & 0.000 \\
$\mathbf{3}$ & 100.000 & 8.515 & 0.000 \\
Mean & 100.000 & 8.560 & 0.000 \\
S.D. & 0.000 & 0.083 & 0.000 \\
$\mathbf{1}$ & & & \\
$\mathbf{2}$ & 100.000 & 16.107 & 0.000 \\
$\mathbf{3}$ & 100.000 & 15.395 & 0.000 \\
Mean & 100.000 & 15.439 & 0.000 \\
S.D. & 100.000 & 15.647 & 0.000 \\
& 0.000 & 0.399 & 0.000
\end{tabular}

\begin{tabular}{|c|c|c|c|}
\hline & \multicolumn{3}{|c|}{ Mole Fraction (x100) } \\
\hline $\mathrm{m} / \mathrm{z}$ & ${ }^{15} \mathrm{~N}_{0}$-PA & ${ }^{15} \mathrm{~N}_{1}-\mathrm{PA}$ & ${ }^{15} \mathrm{~N}_{2}-\mathrm{PA}$ \\
\hline $534->216$ & 92.12 & 0.00 & 0.00 \\
\hline $535->217$ & 7.88 & 92.42 & 0.00 \\
\hline $536->218$ & 0.00 & 7.58 & 92.42 \\
\hline
\end{tabular}

Pseudoinverse Matrix

${ }^{15} \mathrm{~N}_{0}$-PA $\quad{ }^{15} \mathrm{~N}_{1}$-PA $\quad{ }^{15} \mathrm{~N}_{2}$-PA

$\begin{array}{rrrr}\mathbf{5 3 4} \boldsymbol{- >} \mathbf{2 1 6} & 0.0109 & -0.0009 & 0.0001 \\ \mathbf{5 3 5} \boldsymbol{- >} \mathbf{2 1 7} & 0.0000 & 0.0108 & -0.0009 \\ \mathbf{5 3 6} \boldsymbol{- >} \mathbf{2 1 8} & 0.0000 & 0.0000 & 0.0108\end{array}$

Corrected, Tracer:Tracee Ratio (x100)

\begin{tabular}{lrrr} 
& ${ }^{15} \mathrm{~N}_{0}$-PA & ${ }^{15} \mathrm{~N}_{1}$-PA & ${ }^{15} \mathrm{~N}_{2}$-PA \\
\cline { 2 - 4 } $\mathbf{1}$ & 100.00 & -0.05 & 0.00 \\
$\mathbf{2}$ & 100.00 & 0.10 & -0.01 \\
$\mathbf{3}$ & 100.00 & -0.04 & 0.00 \\
Mean & 100.00 & 0.00 & 0.00 \\
S.D. & 0.00 & 0.08 & 0.01 \\
& & & \\
$\mathbf{1}$ & 100.00 & 7.52 & -0.62 \\
$\mathbf{2}$ & 100.00 & 6.81 & -0.56 \\
$\mathbf{3}$ & 100.00 & 6.86 & -0.56 \\
Mean & 100.00 & 7.06 & -0.58 \\
S.D. & 0.00 & 0.40 & 0.03
\end{tabular}

\section{Corrected Isotopic Abundances \& Ratio}

\begin{tabular}{lrr} 
[M] & \multicolumn{1}{c}{ [M+ 1] } & \multicolumn{1}{c}{$[\mathrm{M}+2]$} \\
1.086 & -0.001 & 0.000 \\
1.086 & 0.001 & 0.000 \\
1.086 & 0.000 & 0.000 \\
1.086 & 0.082 & -0.007 \\
1.086 & 0.074 & -0.006 \\
1.086 & 0.074 & -0.006
\end{tabular}


Calculation 3: Presence of $15 \mathrm{~N}$ in the N-terminal Isoleucine Note: Neutral loss isotopic enrichment model

\begin{tabular}{|c|c|c|c|c|c|c|c|}
\hline \multicolumn{4}{|c|}{ Theoretical Isotope Ratio (x100) } & \multicolumn{4}{|c|}{ Mole Fraction (x100) } \\
\hline $\mathrm{m} / \mathrm{z}$ & ${ }^{15} \mathrm{~N}_{0}-\mathrm{PA}$ & ${ }^{15} \mathrm{~N}_{1}-\mathrm{PA}$ & ${ }^{15} \mathrm{~N}_{2}-\mathrm{PA}$ & $\mathrm{m} / \mathrm{z}$ & ${ }^{15} \mathrm{~N}_{0}$-PA & ${ }^{15} \mathrm{~N}_{1}-\mathrm{PA}$ & ${ }^{15} \mathrm{~N}_{2}-\mathrm{PA}$ \\
\hline $534->379$ & 100.00 & 0.00 & 0.00 & $534->379$ & 92.36 & 0.00 & 0.00 \\
\hline $535->379$ & 8.01 & 100.00 & 0.00 & $535->379$ & 7.40 & 92.67 & 0.00 \\
\hline \multirow[t]{5}{*}{$536->379$} & 0.26 & 7.65 & 100.00 & $536->379$ & 0.24 & 7.09 & 92.98 \\
\hline & & 0.26 & 7.29 & & & & \\
\hline & & & 0.26 & & & & \\
\hline & \multicolumn{3}{|c|}{ Isotope Design Matrix } & & \multicolumn{3}{|c|}{ Pseudoinverse Matrix } \\
\hline & ${ }^{15} \mathrm{~N}_{0}-\mathrm{PA}$ & ${ }^{15} \mathrm{~N}_{1}-\mathrm{PA}$ & ${ }^{15} \mathrm{~N}_{2}-\mathrm{PA}$ & & ${ }^{15} \mathrm{~N}_{0}$-PA & ${ }^{15} \mathrm{~N}_{1}$-PA & ${ }^{15} \mathrm{~N}_{2}-\mathrm{PA}$ \\
\hline $534->379$ & 92.361 & 7.395 & 0.244 & $534->379$ & 0.0108 & -0.0009 & 0.0000 \\
\hline $535->379$ & 0.000 & 92.670 & 7.089 & $535->379$ & 0.0000 & 0.0108 & -0.0008 \\
\hline $536->379$ & 0.000 & 0.000 & 92.980 & $536->379$ & 0.0000 & 0.0000 & 0.0108 \\
\hline
\end{tabular}

Sample Measured Isotope Ratio (x100)

\begin{tabular}{lrrr} 
& ${ }^{15} \mathrm{~N}_{0}$-PA & ${ }^{15} \mathrm{~N}_{1}$-PA & ${ }^{15} \mathrm{~N}_{2}$-PA \\
\cline { 2 - 4 } $\mathbf{1}$ & 100.000 & 7.880 & 0.180 \\
$\mathbf{2}$ & 100.000 & 7.621 & 0.283 \\
$\mathbf{3}$ & 100.000 & 8.520 & 0.331 \\
Mean & 100.000 & 8.007 & 0.265 \\
S.D. & 0.000 & 0.463 & 0.077 \\
& & & \\
$\mathbf{1}$ & 100.000 & 10.484 & 0.355 \\
$\mathbf{2}$ & 100.000 & 11.149 & 0.373 \\
$\mathbf{3}$ & 100.000 & 10.290 & 0.457 \\
Mean & 100.000 & 10.641 & 0.395 \\
S.D. & 0.000 & 0.451 & 0.054
\end{tabular}

Corrected, Tracer:Tracee Ratio (x100)

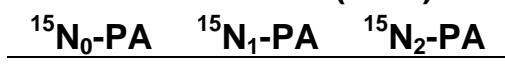

$\begin{array}{rrrrr}\mathbf{1} & & 100.00 & -0.13 & -0.07 \\ \mathbf{2} & & 100.00 & -0.38 & 0.05 \\ \mathbf{3} & & 100.00 & 0.51 & 0.03 \\ \text { Mean } & 100.000 & 0.000 & 0.000 \\ & \text { S.D. } & 0.000 & 0.461 & 0.065\end{array}$

\begin{tabular}{|c|c|c|c|}
\hline 1 & 100.00 & 2.47 & -0.10 \\
\hline 2 & 100.00 & 3.13 & -0.13 \\
\hline 3 & 100.00 & 2.28 & 0.02 \\
\hline Mean & 100.000 & 2.625 & -0.070 \\
\hline S.D. & 0.000 & 0.449 & 0.078 \\
\hline
\end{tabular}

Corrected Isotopic Abundances \& Ratio

$\begin{array}{lrr}\text { [M] } & {[\mathbf{M}+1]} & {[\mathbf{M}+2]} \\ & & \\ 1.083 & -0.001 & -0.001 \\ 1.083 & -0.004 & 0.001 \\ 1.083 & 0.006 & 0.000 \\ 1.083 & 0.027 & -0.001 \\ 1.083 & 0.034 & -0.001 \\ 1.083 & 0.025 & 0.000\end{array}$


Calculation 4: Presence of $15 \mathrm{~N}$ in the central Tyrosine or the AHEP moiety Note: Charged residue isotopic enrichment model

\begin{tabular}{|c|c|c|c|c|c|c|c|}
\hline \multicolumn{4}{|c|}{ Theoretical Isotope Ratio (x100) } & \multicolumn{4}{|c|}{ Mole Fraction (x100) } \\
\hline $\mathbf{m} / \mathbf{z}$ & ${ }^{15} \mathrm{~N}_{0}-\mathrm{PA}$ & ${ }^{15} \mathrm{~N}_{1}-\mathrm{PA}$ & ${ }^{15} \mathrm{~N}_{2}-\mathrm{PA}$ & $\mathrm{m} / \mathrm{z}$ & ${ }^{15} \mathrm{~N}_{0}-\mathrm{PA}$ & ${ }^{15} \mathrm{~N}_{1}-\mathrm{PA}$ & ${ }^{15} \mathrm{~N}_{2}-\mathrm{PA}$ \\
\hline $534->379$ & 100.00 & 0.00 & 0.00 & $534->379$ & 84.21 & 0.00 & 0.00 \\
\hline $535->380$ & 16.89 & 100.00 & 0.00 & $535->380$ & 14.22 & 84.47 & 0.00 \\
\hline \multirow[t]{5}{*}{$536->381$} & 1.86 & 16.53 & 100.00 & $536->381$ & 1.57 & 13.96 & 84.72 \\
\hline & & 1.86 & 16.17 & & & & \\
\hline & & & 1.86 & & & & \\
\hline & \multicolumn{3}{|c|}{ Isotope Design Matrix } & & \multicolumn{3}{|c|}{ Pseudoinverse Matrix } \\
\hline & ${ }^{15} \mathrm{~N}_{0}$-PA & ${ }^{15} \mathrm{~N}_{1}$-PA & ${ }^{15} \mathrm{~N}_{2}-\mathrm{PA}$ & & ${ }^{15} \mathrm{~N}_{0}-\mathrm{PA}$ & ${ }^{15} \mathrm{~N}_{1}-\mathrm{PA}$ & ${ }^{15} \mathrm{~N}_{2}-\mathrm{PA}$ \\
\hline $534->379$ & 84.213 & 14.219 & 1.568 & $534->379$ & 0.0119 & -0.0020 & 0.0001 \\
\hline $535->380$ & 0.000 & 84.467 & 13.962 & $535->380$ & 0.0000 & 0.0118 & -0.0020 \\
\hline $536->381$ & 0.000 & 0.000 & 84.724 & $536->381$ & 0.0000 & 0.0000 & 0.0118 \\
\hline
\end{tabular}

Sample Measured Isotope Ratio (x100)

\begin{tabular}{lrrr} 
& ${ }^{15} \mathrm{~N}_{0}$-PA & ${ }^{15} \mathrm{~N}_{1}$-PA & ${ }^{15} \mathrm{~N}_{2}$-PA \\
\cline { 2 - 4 } $\mathbf{1}$ & 100.000 & 16.567 & 1.841 \\
$\mathbf{2}$ & 100.000 & 16.904 & 1.821 \\
$\mathbf{3}$ & 100.000 & 17.185 & 1.923 \\
Mean & 100.000 & 16.885 & 1.862 \\
S.D. & 0.000 & 0.310 & 0.054 \\
& & & \\
$\mathbf{1}$ & 100.000 & 31.980 & 5.447 \\
$\mathbf{2}$ & 100.000 & 27.237 & 5.079 \\
$\mathbf{3}$ & 100.000 & 29.020 & 5.584 \\
Mean & 100.000 & 29.412 & 5.370 \\
S.D. & 0.000 & 2.396 & 0.261
\end{tabular}

Corrected, Tracer:Tracee Ratio (x100)

\begin{tabular}{lrrr} 
& ${ }^{15} \mathbf{N}_{0}-\mathbf{P A}$ & ${ }^{15} \mathbf{N}_{1}$-PA & ${ }^{15} \mathbf{N}_{2}$-PA \\
\cline { 2 - 4 } $\mathbf{1}$ & 100.00 & -0.32 & 0.03 \\
$\mathbf{2}$ & 100.00 & 0.02 & -0.04 \\
$\mathbf{3}$ & 100.00 & 0.30 & 0.01 \\
Mean & 100.000 & 0.000 & 0.000 \\
S.D. & 0.000 & 0.309 & 0.039
\end{tabular}

\begin{tabular}{|rrrr|}
\hline $\mathbf{1}$ & 100.00 & 15.05 & 1.08 \\
$\mathbf{2}$ & 100.00 & 10.32 & 1.50 \\
$\mathbf{3}$ & 100.00 & 12.10 & 1.71 \\
\hline Mean & 100.000 & 12.489 & 1.429 \\
\hline S.D. & 0.000 & 2.388 & 0.317 \\
\hline
\end{tabular}

Corrected Isotopic Abundances \& Ratio

\begin{tabular}{lrr} 
[M] & \multicolumn{1}{l}{ [M + 1] } & \multicolumn{1}{c}{$[\mathrm{M}+2]$} \\
& & \\
1.187 & -0.004 & 0.000 \\
1.187 & 0.000 & -0.001 \\
1.187 & 0.004 & 0.000 \\
1.187 & 0.179 & 0.013 \\
1.187 & 0.123 & 0.018 \\
1.187 & 0.144 & 0.020
\end{tabular}



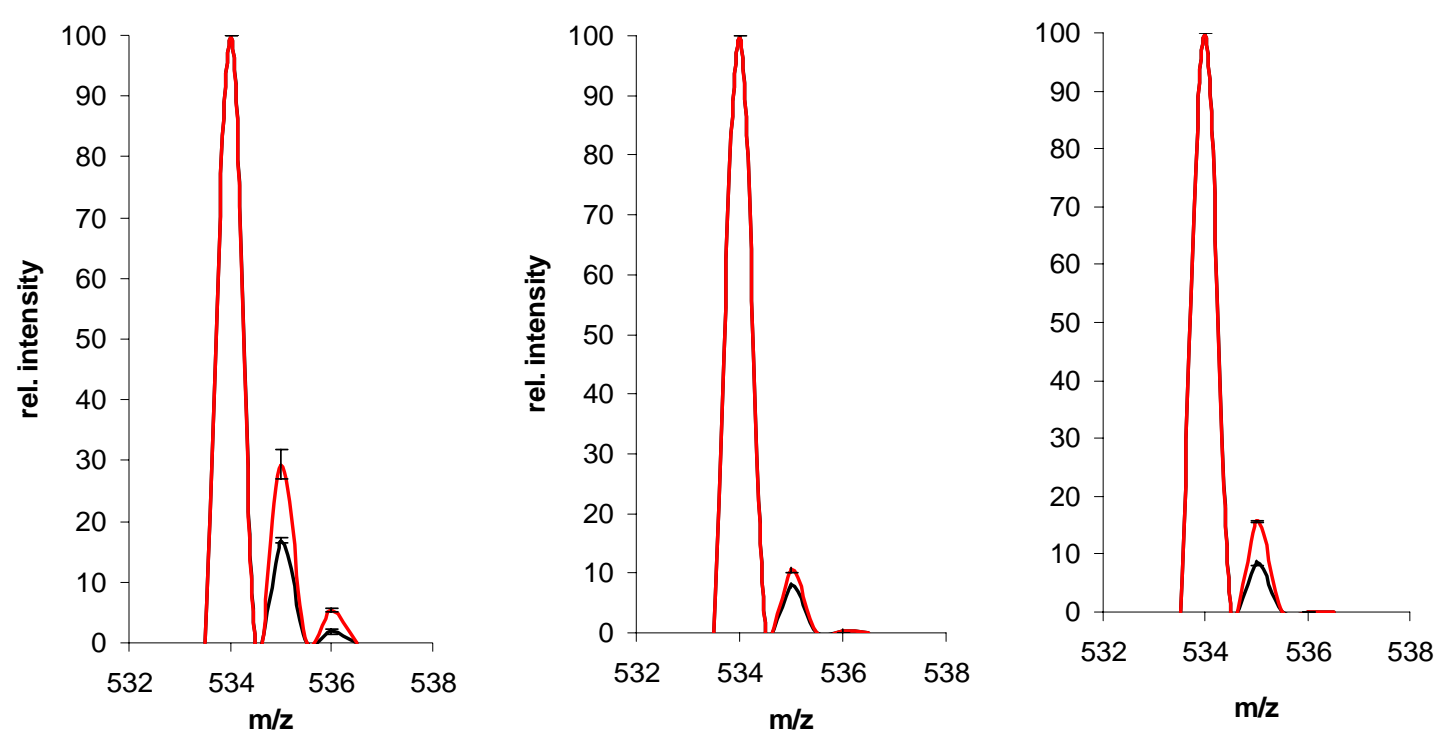

Figure 6. Simulated isotopic distributions based on least squares fitting of isotopomer data in Calculations 1-4. Red is labeled black is unlabeled. Left: $\%{ }^{15} \mathrm{~N}$ enrichment in Tyr-AHEP fragment. Middle: $\%{ }^{15} \mathrm{~N}$ enrichment in N-Ac-Ile fragment. Right: $\%{ }^{15} \mathrm{~N}$ in AHEP alone.

$\boldsymbol{d}_{\mathbf{2}}$-tyrosine. The mass spectrometer was operated in the positive ion mode and the electrospray needle was maintained at $4200 \mathrm{~V}$. The ion transfer tube was operated at $35 \mathrm{~V}$ and $300^{\circ} \mathrm{C}$. The tube lens voltage was set to $131 \mathrm{~V}$. Source CID (offset voltage between skimmer and the first ion quide, Q00) was used at 15V. The selected reaction monitoring (SRM) mode was used. Ions were collisionally activated with argon at an indicated pressure of $1.5 \mathrm{mT}$. The mass-spectral resolution was set to a peak width (full width at half maximum, FWHM) of 0.50u and $0.50 \mathrm{u}$ for precursor and product ions respectively. Mass transitions at the specified collision energy $(\mathrm{m} / \mathrm{z} 536 \rightarrow 218 ; 20 \mathrm{eV}),(\mathrm{m} / \mathrm{z} 537 \rightarrow 219 ; 20 \mathrm{eV}),(\mathrm{m} / \mathrm{z} 538 \rightarrow 220 ; 20 \mathrm{eV})$, and $(\mathrm{m} / \mathrm{z} 539 \rightarrow 221 ; 20 \mathrm{eV})$ were monitored for AHEP, AHEP+1, AHEP+2, and AHEP+3, respectively. Additionally, transitions at the specified collision energy ( $\mathrm{m} / \mathrm{z} 536 \rightarrow 381 ; 20 \mathrm{eV})$, $(\mathrm{m} / \mathrm{z}$ 537 $\rightarrow 382 ; 20 \mathrm{eV}),(\mathrm{m} / \mathrm{z} 538 \rightarrow 383 ; 20 \mathrm{eV}),(\mathrm{m} / \mathrm{z} 539 \rightarrow 384 ; 20 \mathrm{eV})$, and $(\mathrm{m} / \mathrm{z} 540 \rightarrow 385$; 
$20 \mathrm{eV}$ ) were monitored for Tyr+AHEP, Tyr+AHEP+1, Tyr+AHEP+2, Tyr+AHEP+3, and Tyr+AHEP+4, respectively. The scan width for product ions was $1.000 \mathrm{u}$ and the cycle time for each ion was 0.15 seconds. The electron multiplier gain was set to $2 \times 10^{6}$. Data were acquired in profile mode. The resulting chromatograms are illustrated in figures 7 and 8 . Both a $\mathrm{d}_{2^{-}}$ tyrosine enriched sample and an unenriched sample were scanned. The unenriched sample was used to create a least squares curve for the data. The same method of calculation as in the ${ }^{15} \mathrm{~N}$ tyrosine experiment was used for determining the amount and position of incorporation. The enrichment level in the central tyrosine was calculated by subtracting the AHEP enrichment from the Tyr-AHEP enrichment. Simulated isotopic distributions based on least squares fitting of isotopomer data are shown in figure 9 .

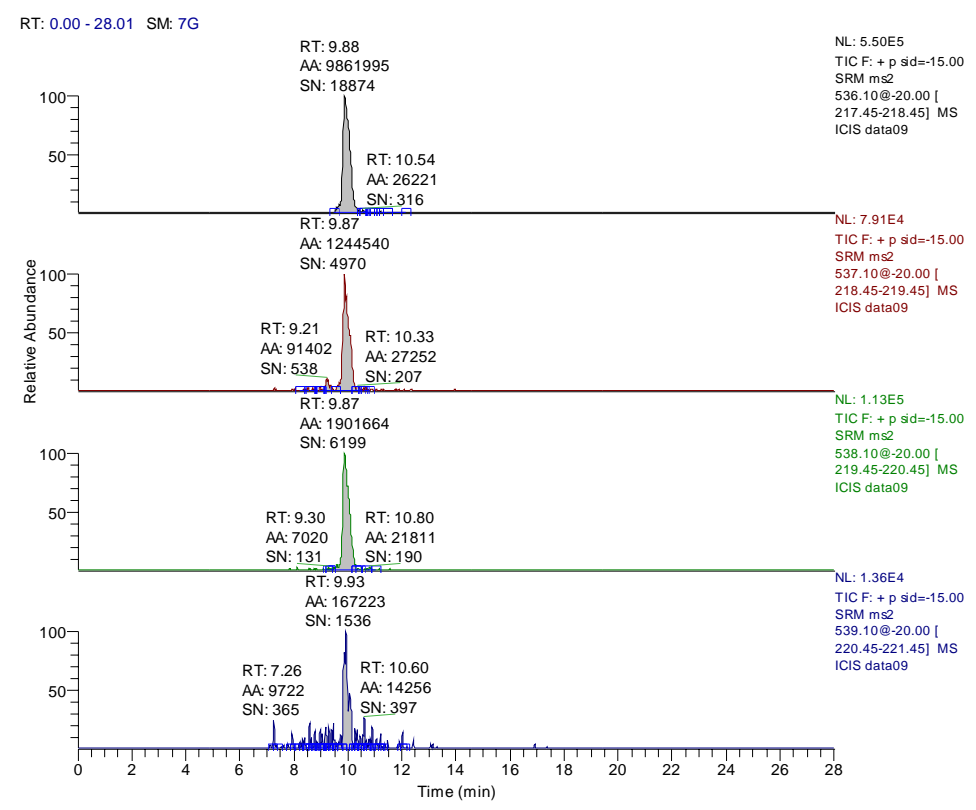

Figure 7. Chromatograms used in the calculation of 3,3- $d_{2}$-tyrosine incorporation in AHEP fragment. 


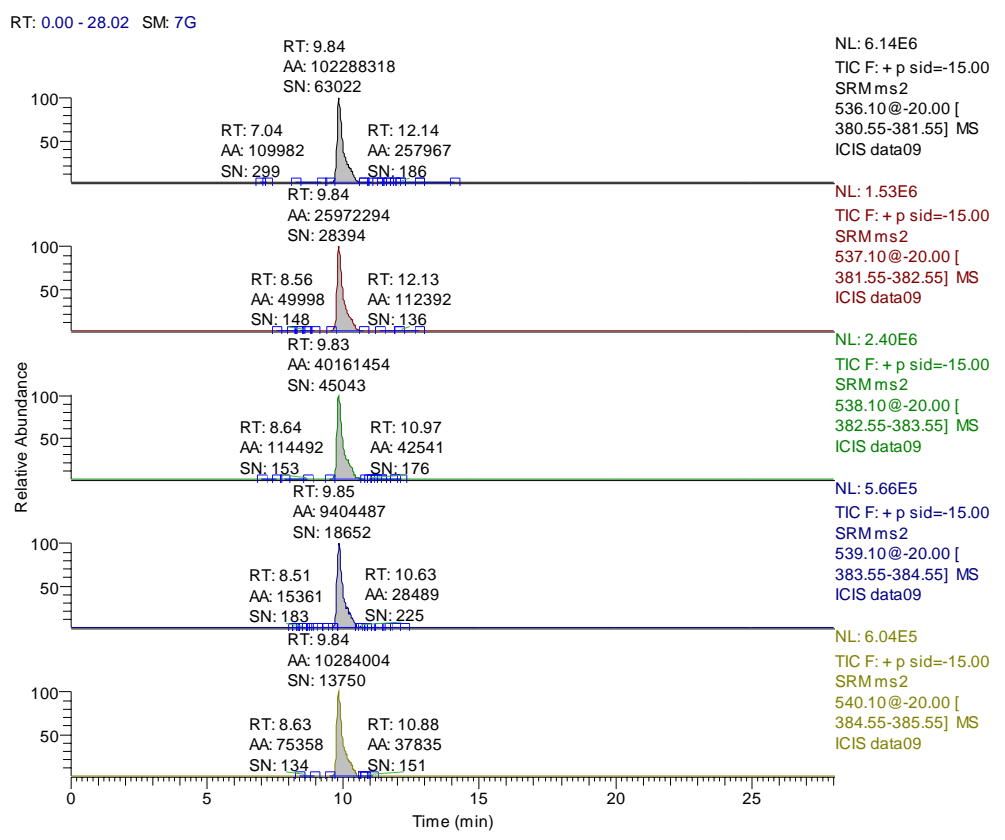

Figure 8. Chromatograms used in the calculation of 3,3- $d_{2}$-tyrosine incorporation in TyrAHEP dipeptide fragment.
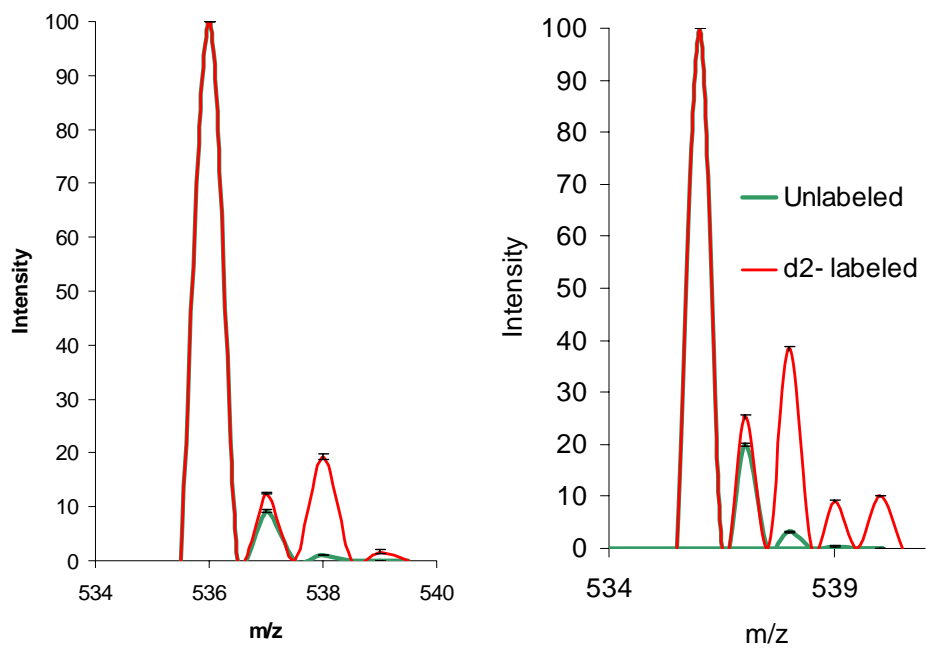

Figure 9. Simulated isotopic distributions based on least squares fitting of isotopomer data from chromatograms in figure 7 and 8 . Red is labeled sample, green is unlabeled. Left: calculated $\% \beta-d_{2}$-Tyrosine in AHEP fragment. These data clearly show conservation of both deuteriums into AHEP and very little evidence of loss of single deuterium. Right: $\% \beta-d_{2^{-}}$ Tyrosine in Tyr-AHEP fragment confirming double incorporation. 